\title{
EGU21-9695
}

https://doi.org/10.5194/egusphere-egu21-9695

EGU General Assembly 2021

(c) Author(s) 2022. This work is distributed under

the Creative Commons Attribution 4.0 License.

\section{Complex rotated CCA: a method to correlate lagged geophysical variables}

\author{
Niclas Rieger ${ }^{1,2}$, Alvaro Corral ${ }^{1}$, Antonio Turiel ${ }^{2}$, and Estrella Olmedo ${ }^{2}$ \\ ${ }^{1}$ Centre for Mathematical Research (CRM), Barcelona, Spain \\ ${ }^{2}$ Institute of Marine Sciences (ICM-CSIC), Barcelona, Spain
}

The nature of the climate system is very complex: a network of mutual interactions between ocean and atmosphere lead to a multitude of overlapping geophysical processes. As a consequence, the same process has often a signature on different climate variables but with spatial and temporal shifts. Orthogonal decompositions, such as Canonical Correlation Analysis (CCA), of geophysical data fields allow to filter out common dominant patterns between two different variables by maximizing cross-correlation. In general, however, CCA suffers from (i) the orthogonality constraint, which tends to produce unphysical patterns, and (ii) the use of direct correlations, which leads to signals that are merely shifted in time being considered as distinct patterns.

In this work, we propose an extension of CCA, complex rotated CCA (crCCA), to address both limitations. First, we generate complex signals by using the Hilbert transforms. To reduce the spatial leakage inherent in Hilbert transforms, we extend the time series using the Theta model, thus creating an anti-leakage buffer space. We then perform the orthogonal decomposition in complex space, allowing us to detect out-of-phase signals. Subsequent Varimax rotation removes the orthogonal constraints to allow more geophysically meaningful modes.

We applied crCCA to a pair of variables expected to be coupled: Pacific sea surface temperature and continental precipitation. We show that crCCA successfully captures the temporally and spatially complex modes of (i) seasonal cycle, (ii) canonical ENSO, and (iii) ENSO Modoki, in a compact manner that allows an easy geophysical interpretation. The proposed method has the potential to be useful especially, but not limited to, studies on the prediction of continental precipitation by other climate variables. An implementation of the method is readily available as a Python package. 